\section{Evaluación de las características microbiológicas y fisicoquímicas del agua sometida a procesos de esterilización en autoclaves a vapor}

\author{
assessment of microbiological and physicochemical characteristics of water sub- \\ jected to sterilization processes in steam autoclaves
}

\author{
Johanna Tapias-Rivera ${ }^{1}$, Christian Chacin-Zambrano², Oscar Guarín-Villamizar ${ }^{3}$, Juan Carlos Uribe-Caputi ${ }^{4}$
}

Forma de citar: J. Tapias-Rivera, C. Chacin-Zambrano, O. Guarín-Villamizar, J.C. Uribe-Caputi, "Evaluación de las características microbiológicas y fisicoquímicas del agua sometida a procesos de esterilización en autoclaves a vapor”, Respuestas, vol. 22, no. 1, pp. 59-66, 2017.

Recibido:

Enero 26 de 2017

Aceptado:

Junio 2 de 2017
'Especialista en epidemiologia, Johanna.tapias@udes.edu.co Orcid: 0000-0001-9353-7904

Profesor Asistente Universidad de Santander, Bucaramanga-

Colombia.

${ }^{2}$ Magister en biotecnología microbiana, cchacin@udes. edu.co Orcid: 0000-00017241-3822, Profesor Asociado

Universidad de Santander, Bucaramanga-Colombia.

${ }^{3}$ Magister en Gestión y auditorías ambientales oguarin@udes.edu.co Orcid: 0000-0003-4496-9932

Profesor Titular Universidad de Santander, BucaramangaColombia.

${ }^{4}$ Magister en epidemiologia jcuribe@gmail.com Orcid: 0000 $00024971339 \mathrm{X}$

Profesor Asistente Universidad de Santander, BucaramangaColombia

\section{Resumen}

El presente trabajo describe las características fisicoquímicas y microbiológicas del agua antes y después de ser sometidas a un proceso de esterilización en un autoclave a vapor con carga de instrumental y paquetes de vestuario quirúrgico, con el fin de establecer el potencial para el posterior sin causar ningún efecto en la validez de la carga por ciclo. Las mediciones fueron descritas por separado, antes del primer proceso de esterilización, durante la etapa de descarga y de la entrada del agua que fue utilizada para dicho proceso. Para el desarrollo del proceso, se realizaron tres tomas de muestras con agua de osmosis y 5 correspondientes al agua directamente de grifo; posterior a cada toma de muestra se tomaron en cuenta los parámetros de esterilización, resoluciones ambientales y microbiológicas. Los resultados obtenidos mostraron significancia en los diferentes puntos de toma de muestras que llevaron a concluir alteraciones en el agua de osmosis en los diferentes puntos de muestra (entrada, chaqueta y descarga) del autoclave en lo relacionado a las características fisicoquímicas del agua obtenida de los puntos analizados del autoclave. Adicionalmente se observó presencia de coliformes totales y fecales, aerobios mesófilos que indicaron que el agua no es apta para recirculación dentro del autoclave.

Palabras clave: agua, autoclave, esterilización, vapor.

\begin{abstract}
This study describes the physicochemical and microbiological characteristics of the water before and after being subjected to sterilization process in steam autoclave loaded with instrumental and surgical clothes, in order to establish its potential use without causing any effect on the validity of the charging cycle. Measurements were described separately before the first sterilization process, during the discharge stage and at the entry of water that was used for this process. For the development of the process, three samples were taken with osmosis water and five directly from tap water; after each sample the parameters of sterilization, and environmental and microbiological resolutions were taken into account. The results showed significance in the different sampling points leading to conclude disturbances in the osmosis water at different sampling points (input, jacket and discharge) of the autoclave regarding the physicochemical characteristics of water obtained from the points analyzed in the autoclave. Additionally, presence of total and fecal coliforms was
\end{abstract}


No. 2

Julio - Dic. 2017

ISSN 0122-820X

E-ISSN 2422-5053

PP: 59-66

\section{Introducción}

Los autoclaves de vapor son las más utilizadas en la industria de centrales hospitalarias, debido a que permiten la esterilización de material que soporte temperaturas entre $121^{\circ}$ y $134^{\circ} \mathrm{C}$, además de los costos en comparación con otros y el tiempo de exposición; Sin embargo, en cada ciclo en el que se utilice observed as well as aerobic mesophilic bacteria indicating that water is not suitable for recirculation within the autoclave

Keywords: water, autoclave, sterilization, steam.

\section{Resumo}

O presente trabalho descreve as características físico-químicas e microbiológicas da água antes e depois de ser submetido a um processo de esterilização em uma autoclave de vapor carregada com instrumentos e embalagens de vestuário cirúrgico, a fim de estabelecer o potencial para o posterior sem causar qualquer efeito sobre a validade da carga por ciclo. As medidas foram descritas separadamente, antes do primeiro processo de esterilização, durante a descarga e a fase de entrada da água que foi utilizada para o referido processo. Para o desenvolvimento do processo, foram colhidas três amostras com água de osmose e 5 correspondentes à água diretamente da torneira; Após cada amostragem, foram considerados os parâmetros de esterilização, as resoluções ambientais e microbiológicas. Os resultados obtidos mostraram diferenças significativas nos pontos de amostragem que levaram à conclusão de alterações na água de osmose nos diferentes pontos de amostra (entrada, revestimento e descarga) da autoclave em relação às características físicoquímicas da água obtida das amostras. pontos da autoclave. Além disso, observou-se a presença de coliformes totais e fecais, mesófilos aeróbicos que indicaram que a água não é adequada para a recirculação dentro da autoclave.

Palavras-chave: Água, autoclave, esterilização, vapor. se gastan aproximadamente entre 25 y 35 litros de agua dependiendo de la capacidad del mismo.[1], [2] Todo esto llevo a centrar la mirada hacia un horizonte que permita evaluar el agua de los autoclaves utilizada en cada proceso de esterilización manteniendo sus propiedades microbiológicas y fisicoquímicas en un número determinado de ciclos sin afectar los resultados del proceso de esterilización de material médico-quirúrgico, los resultados obtenidos representarían un gran beneficio no solo institucional (económico), sino también ambiental [3]. La esterilización a vapor es un proceso físico que se fundamenta en la coagulación de estructuras proteicas en los microorganismos y resulta ser el de elección para materiales que toleran temperaturas de proceso de entre $121^{\circ} \mathrm{C} / 134^{\circ} \mathrm{C}$, se usa para esterilizar materiales termoestables y no sensibles a la humedad [4]. Es ampliamente utilizado en instrumental quirúrgico, textiles, materiales de vidrio boro silicato, papel, cerámica, medios de cultivo, prótesis e implantes, entre otros [5] Este proceso ocurre a través de una autoclave, el cual es un recipiente metálico de paredes gruesas con cierre hermético que permite trabajar con vapor de agua a alta presión y alta temperatura. Es muy importante que el vapor sea limpio y puro ante estos requerimientos y características del agua necesarias para un 
ciclo de esterilización, se puede evidenciar que habrá aproximadamente 25 litros del líquido por ciclo, que una vez terminado se desecharan por las tuberías, lo que genera gran impacto para el medio ambiente. Razón por la cual se considera importante la evaluación de las características microbiológicas y fisicoquímicas del agua sometida a procesos de esterilización en autoclaves a vapor [6].

\section{Materiales Y Métodos}

\section{Procedimiento}

El estudio se realizó en una empresa fabricante de autoclaves a vapor en la ciudad de Bucaramanga donde se contó con los equipos requeridos para la investigación. El trabajo de campo se desarrolló entre los meses mayo y junio del 2015 con personal de ingeniería ambiental, microbiología industrial e instrumentación quirúrgica. Los tres puntos de toma de muestra del agua que se establecieron fueron el calderin, descarga y entrada del agua. A partir de ello, se realizaron los análisis microbiológicos y fisicoquímicos.

\section{Análisis Microbiológico}

A partir de los $150 \mathrm{~mL}$ de muestra de cada uno de los puntos, se realizaron los siguientes análisis: Recuento de Microorganismos Aerobios Mesófilos, Clostridium Sulfito Reductor (Presencia - Ausencia), Mohos y Levaduras, Coliformes fecales y totales y Bacillus subtilis. Los resultados obtenidos se analizaron de acuerdo a la resolución 2115 de 2007 (Resolucion 2115, 2007), NTC 813 (NTC 813, 2007), ISO 8199 del 2008 (ISO 4833 - aerobios mesófilos, ISO 93081 - coliformes totales y fecales, ISO 6461 Clostridium Sulfito Reductor) y (Toro, 2013).

\section{Análisis Físico-químico}

A partir del volumen establecido para cada punto de toma de muestras, se realizaron los siguientes análisis físico-químicos: Caudal, pH. Temperatura, DQO, Turbidez, Color y Conductividad. Los resultados obtenidos se analizaron de acuerdo a la resolución 2115 de 2007, Decreto 1541 de 1978, Decreto 1594 de 1984, Decreto 475 de 1998.

\section{Resultados}

De acuerdo al estudio, se obtuvieron los siguientes resultados, descritos en la tabla I:

Tabla I. Resultado análisis fisicoquímico y microbiológico de la autoclave

\begin{tabular}{|c|c|c|c|c|c|c|c|}
\hline PARÁMETROS & $\begin{array}{c}\text { PUNTO 1. } \\
\text { CALDERÍN } \\
\text { AGUA } \\
\text { ÓSMOSIS }\end{array}$ & $\begin{array}{c}\text { PUNTO } 2 . \\
\text { DESCARGA } \\
\text { ÓSMOSIS }\end{array}$ & $\begin{array}{l}\text { PUNTO } 3 . \\
\text { ÓSMOSIS }\end{array}$ & $\begin{array}{c}\text { PUNTO } 4 . \\
\text { CALDERÍN } \\
\text { AGUA GRIFO }\end{array}$ & $\begin{array}{c}\text { PUNTO 5. } \\
\text { DESCARGA } \\
\text { AGUA } \\
\text { GRIFO }\end{array}$ & $\begin{array}{c}\text { PUNTO } 6 . \\
\text { AGUA } \\
\text { GRIFO }\end{array}$ & $\begin{array}{c}\text { NORMATIVIDAD } \\
\text { AMBIENTAL }\end{array}$ \\
\hline MUESTRA & $100 \mathrm{~mL}$ & $40 \mathrm{~mL}$ & $100 \mathrm{~mL}$ & $150 \mathrm{~mL}$ & $50 \mathrm{~mL}$ & $500 \mathrm{~mL}$ & - \\
\hline CAUDAL & $1,89 \times 10^{-3} \mathrm{~L} / \mathrm{s}$ & $2,28 \times 10^{-3} \mathrm{~L} / \mathrm{s}$ & $0,02 \mathrm{~L} / \mathrm{s}$ & $2,11 \times 10^{-3} \mathrm{~L} / \mathrm{s}$ & $8,33 \times 10^{-3} \mathrm{~L} / \mathrm{s}$ & $0,05 \mathrm{~L} / \mathrm{s}$ & $1.5(\mathrm{QMH})$ \\
\hline pH IN SITU & 4 & 7 & 3 & 5 & 6 & 7 & \multirow[b]{2}{*}{$5-9$ unidades } \\
\hline $\begin{array}{c}\text { pH } \\
\text { LABORATORIO } \\
\end{array}$ & 4 & 6 & 4 & 5 & 6 & 7 & \\
\hline TEMPERATURA & $81^{\circ} \mathrm{C}$ & $62{ }^{\circ} \mathrm{C}$ & $25^{\circ} \mathrm{C}$ & $81{ }^{\circ} \mathrm{C}$ & $60^{\circ} \mathrm{C}$ & $28^{\circ} \mathrm{C}$ & $<40^{\circ} \mathrm{C} * *$ \\
\hline DQO & $43 \mathrm{mg} / \mathrm{L}$ & $265 \mathrm{mg} / \mathrm{L}$ & $12 \mathrm{mg} / \mathrm{L}$ & $7 \mathrm{mg} / \mathrm{L}$ & $273 \mathrm{mg} / \mathrm{L}$ & $12 \mathrm{mg} / \mathrm{L}$ & $200 \mathrm{mg} / \mathrm{L}$ \\
\hline TURBIDEZ & $0,49 \mathrm{NTU}$ & $65,4 \mathrm{NTU}$ & $0,39 \mathrm{NTU}$ & $1,06 \mathrm{NTU}$ & $30 \mathrm{NTU}$ & $\begin{array}{l}\mathrm{N} \\
\mathrm{T} \\
\mathrm{U}\end{array}$ & $<5$ unidades \\
\hline COLOR & $3 \mathrm{PtCo}$ & $122 \mathrm{PtCo}$ & * & $15 \mathrm{PtCo}$ & $371{ }^{\circ} \mathrm{C}$ & $5 \mathrm{PtCo}$ & 75 unidades \\
\hline $\begin{array}{c}\text { CONDUCTIVIDA } \\
\text { D } \\
\end{array}$ & $450,5 \mu \mathrm{S} / \mathrm{cm}$ & $217,1 \mu \mathrm{S} / \mathrm{cm}$ & * & $126,9 \mu \mathrm{S} / \mathrm{cm}$ & $65,20 \mu \mathrm{S} / \mathrm{cm}$ & $\begin{array}{l}212,4 \\
\mu \mathrm{S} / \mathrm{cm}\end{array}$ & $<1500 \mu \mathrm{S} / \mathrm{cm}$ \\
\hline $\begin{array}{c}\text { AEROBIOS } \\
\text { MESOFILOS }\end{array}$ & Incontables & $0 \mathrm{ufc}$ & $0 \mathrm{ufc}$ & 0 ufc & $0 \mathrm{ufc}$ & 0 ufc & $100 \mathrm{UFC} / 100 \mathrm{~mL}$ \\
\hline Clostridium & $0 \mathrm{ufc}$ & $0 \mathrm{ufc}$ & $0 \mathrm{ufc}$ & $0 \mathrm{ufc}$ & $0 \mathrm{ufc}$ & 0 ufc & $0 \mathrm{UFC} / 100 \mathrm{~mL}$ \\
\hline $\begin{array}{c}\text { HONGOS Y } \\
\text { LEVADURAS }\end{array}$ & $0 \mathrm{ufc}$ & $0 \mathrm{ufc}$ & $37 \mathrm{ufc}$ & 0 ufc & $0 \mathrm{ufc}$ & $0 \mathrm{ufc}$ & $20 \mathrm{UFC} / 100 \mathrm{~mL}$ \\
\hline $\begin{array}{c}\text { COLIFORMES } \\
\text { FECALES Y } \\
\text { TOTALES } \\
\end{array}$ & $2 \mathrm{ufc}$ & $0 \mathrm{ufc}$ & $0 \mathrm{ufc}$ & $0 \mathrm{ufc}$ & $0 \mathrm{ufc}$ & $0 \mathrm{ufc}$ & $0 \mathrm{UFC} / 100 \mathrm{~mL}$ \\
\hline
\end{tabular}


De acuerdo al Decreto 3930/2010, se evidencia que los parámetros evaluados sobrepasan a los establecidos como normales. El Decreto ordena que todo vertimiento a un cuerpo de agua deberá cumplir, por lo menos, con las siguientes normas: Referencia Usuario Existente Usuario Nuevo un $\mathrm{pH}$ 5 a 9 unidades y una Temperatura $<40^{\circ} \mathrm{C}$ $<40^{\circ} \mathrm{C}$ Demanda bioquímica de oxígeno: Para desechos domésticos Remoción $>30 \%$ en carga Remoción $>80 \%$ en carga Para desechos industriales Remoción $>20 \%$ en carga Remoción $>80 \%$ en carga, Color real 75 unidades, escala. Lo que indica de acuerdo con la tabla de resultados que el $\mathrm{pH}$ in situ tomado específicamente en la autoclave y de laboratorio llevado a cabo luego de 24 horas, son menores en el punto calderin agua osmosis y el punto tres de osmosis; la temperatura es muy alta sobrepasando los parámetros normales en el punto calderin, descarga y entrada de agua de osmosis, en los puntos entrada y descarga agua de grifo y el Color sobrepasa los niveles en los puntos descarga agua grifo y osmosis. De acuerdo a la Resolución 0631 Artículo 8 Página 7 Valores límites máximos permisibles en los vertimientos puntuales de ARD de las soluciones individuales de saneamiento de viviendas unifamiliares o bifamiliares. La demanda química de oxigeno DQO con unidades en $\mathrm{mg} / \mathrm{L} \mathrm{O}_{2}$ debe ser hasta un límite de 200,00; la tabla de resultados muestra que los puntos de descarga osmosis y descarga de agua grifo sobrepasan el nivel máximo normal. Según el Decreto 475/98 Artículo 8 Literal b) y Artículo 36 Criterios químicos de la calidad de agua potable y criterios de calidad organolépticas y físicas del agua segura la turbidez nefelometrica debe ser $<5$ turbidez UNT y la conductividad micro siemens $/ \mathrm{cm}$ $<1.500$ lo que indica que en la tabla en los puntos descarga osmosis y descarga grifo la turbidez sobrepasan los niveles vigentes normales ambientales. El resto de parámetros se muestras en niveles normales según grafica de la primera toma de muestra y teniendo en cuenta la norma ambiental vigente.

Según Resolución 2115 de 2007 Articulo 11 Características microbiológicas y en el parágrafo 1. Las características microbiológicas del agua para consumo humano deben enmarcarse dentro de los valores de 1 Unidad Formadora de Colonia (UFC) ó 1 microorganismo en $100 \mathrm{~cm}^{3} \mathrm{de}$ muestra, De acuerdo a los resultados arrojados en la toma de muestra que se observan en la tabla los parámetros afectados y que se encuentran fuera de lo normal es los aerobios mesófilos y los coliformes fecales y totales. Y según la Norma Técnica Colombiana 813 (segunda actualización) el número de colonias producido por los hongos o levaduras no debe exceder de 20 colonias (U.F.C) en $100 \mathrm{~cm} 3$ independientemente del método de análisis realizado a la muestra, ninguna debe contener presencia de estos microorganismo. En base a los resultados obtenidos en el punto de descarga sobrepaso la normatividad lo cual requirió examinar ese punto en las pruebas siguientes hasta confirmar la ausencia de estos microorganismos (ver tabla II). 
Tabla II. Resultados análisis fisicoquímico única toma manual de muestras simples en autoclave de una institución de salud.

\begin{tabular}{|c|c|c|c|c|c|c|c|}
\hline PARÁMETROS & $\begin{array}{l}\text { PUNTO } 1 . \\
\text { ENTRADA } \\
\text { ÓSMOSIS }\end{array}$ & $\begin{array}{c}\text { PUNTO } 2 . \\
\text { ÓSMOSIS } \\
\text { CHAQUETA }\end{array}$ & $\begin{array}{c}\text { PUNTO } 3 . \\
\text { ENTRADA } \\
\text { GRIFO }\end{array}$ & $\begin{array}{l}\text { PUNTO } 4 . \\
\text { DESCARGA } \\
\text { ÓSMOSIS }\end{array}$ & $\begin{array}{l}\text { PUNTO } 5 . \\
\text { DESCARGA } \\
\text { GRIFO }\end{array}$ & $\begin{array}{c}\text { PUNTO } 6 . \\
\text { DESCARGA } \\
\text { ÓSMOSIS* }\end{array}$ & $\begin{array}{l}\text { NORMATIVIDA } \\
\text { D AMBIENTAL }\end{array}$ \\
\hline MUESTRA & $100 \mathrm{~mL}$ & $120 \mathrm{~mL}$ & $120 \mathrm{~mL}$ & $20 \mathrm{~mL}$ & $40 \mathrm{~mL}$ & $80 \mathrm{~mL}$ & - \\
\hline $\begin{array}{c}\mathrm{pH} \\
\text { LABORATORIO }\end{array}$ & 6,87 & 6,89 & 6,80 & 6,90 & 6,90 & 6,90 & $5-9$ \\
\hline DQO & 26 & 28 & 124 & 170 & 99 & 219 & $200 \mathrm{mg} / \mathrm{L}$ \\
\hline TURBIDEZ & $0,81 \mathrm{NTU}$ & $0,77 \mathrm{NTU}$ & $1,20 \mathrm{NTU}$ & 11,2 NTU & 7,09 NTU & $11,6 \mathrm{NTU}$ & $<5$ unidades \\
\hline COLOR & $29 \mathrm{PtCo}$ & $25 \mathrm{PtCo}$ & $55 \mathrm{PtCo}$ & $168 \mathrm{PtCo}$ & $109 \mathrm{PtCo}$ & $144 \mathrm{PtCo}$ & 75 unidades \\
\hline $\begin{array}{c}\text { CONDUCTIVID } \\
\text { AD }\end{array}$ & $16,45 \mu \mathrm{S} / \mathrm{cm}$ & $5,69 \mu \mathrm{S} / \mathrm{cm}$ & $136,4 \mu \mathrm{S} / \mathrm{cm}$ & $26,32 \mu \mathrm{S} / \mathrm{cm}$ & $33,79 \mu \mathrm{S} / \mathrm{cm}$ & $14,54 \mu \mathrm{S} / \mathrm{cm}$ & $<1500 \mu \mathrm{S} / \mathrm{cm}$ \\
\hline ALCALINIDAD & $\begin{array}{c}4 \text { gotas x } 20 \\
80 \mathrm{mg} / \mathrm{L}\end{array}$ & $\begin{array}{c}\text { 3gotas x } 20 \\
60 \mathrm{mg} / \mathrm{L} \\
\end{array}$ & $\begin{array}{c}11 \text { gotas x } 20 \\
220 \mathrm{mg} / \mathrm{L}\end{array}$ & -- & --- & $\begin{array}{c}2 \text { gotas x } 20 \\
40 \mathrm{mg} / \mathrm{L}\end{array}$ & $100 \mathrm{mg} / \mathrm{L}$ \\
\hline DUREZA & $3 \mathrm{ppm}$ & $16 \mathrm{ppm}$ & $14 \mathrm{ppm}$ & -- & --- & $\geq 100 \mathrm{ppm}$ & $160 \mathrm{mg} / \mathrm{L}$ \\
\hline \multicolumn{8}{|l|}{$\begin{array}{l}\text { CARBONO } \\
\text { ORGÁNICO } \\
\text { TOTAL } \\
\end{array}$} \\
\hline $\begin{array}{c}\text { AEROBIOS } \\
\text { MESOFILOS } \\
\end{array}$ & 250 ufc & 0 ufc & 0 ufc & $0 \mathrm{ufc}$ & $0 \mathrm{ufc}$ & $0 \mathrm{ufc}$ & $100 \mathrm{UFC} / 100 \mathrm{~mL}$ \\
\hline Clostridium & 0 ufc & $0 \mathrm{ufc}$ & 0 ufc & $0 \mathrm{ufc}$ & 0 ufc & $0 \mathrm{ufc}$ & $0 \mathrm{UFC} / 100 \mathrm{~mL}$ \\
\hline $\begin{array}{c}\text { HONGOS Y } \\
\text { LEVADURAS }\end{array}$ & 0 ufc & 0 ufc & 0 ufc & $0 \mathrm{ufc}$ & 0 ufc & $0 \mathrm{ufc}$ & $20 \mathrm{UFC} / 100 \mathrm{~mL}$ \\
\hline $\begin{array}{c}\text { COLIFORMES } \\
\text { FECALES Y } \\
\text { TOTALES } \\
\end{array}$ & 0 ufc & 0 ufc & 0 ufc & 0 ufc & 0 ufc & 0 ufc & $0 \mathrm{UFC} / 100 \mathrm{~mL}$ \\
\hline Bacillus sp & Ausencia & Ausencia & Ausencia & Ausencia & Ausencia & Ausencia & ausentes \\
\hline
\end{tabular}

Fuente: Autores

De acuerdo a las tomas de agua osmosis y grifo en la institución de salud, los resultados según la Resolución 0631 Artículo 8 Página 7 Valores límites máximos permisibles en los vertimientos puntuales de ARD de las soluciones individuales de saneamiento de viviendas unifamiliares o bifamiliares. La demanda química de oxigeno DQO con unidades en $\mathrm{mg} / \mathrm{L} \mathrm{O} 2$ debe ser hasta un límite de 200,00; la tabla de resultados muestra que los puntos de descarga agua osmosis sobrepasan el nivel máximo normal. Según el Decreto 475/98 Artículo 8 Literal b) y Artículo 36 Criterios químicos de la calidad de agua potable y criterios de calidad organolépticas y físicas del agua segura la turbidez nefelométrica debe ser $<5$ turbidez UNT y la conductividad micro siemens/cm $<1.500$ lo que indica que en la tabla en los puntos descarga grifo, osmosis la turbidez sobrepasan los niveles vigentes normales ambientales. De acuerdo a los resultados visibles y especificados en la tabla los valores normales según al Decreto 1594/84 Artículos 38,72 y 73 . Vigente aun porque el Decreto 3930/2010 empezará a ser vigente a partir de enero de 2016, sobrepasa los parámetros normales ya que el decreto ordena Todo vertimiento a un cuerpo de agua deberá cumplir, por lo menos, con las siguientes norma: Color real 75 unidades, escala. Lo que indica de acuerdo a la tabla de resultados el Color no obedece a los niveles en los puntos descarga agua grifo y osmosis. El resto de parámetros se muestras en niveles normales según grafica de la primera toma de muestra y teniendo en cuenta la norma ambiental vigente.

Según Resolución 2115 de 2007 Articulo 11 parágrafo 1. Las características microbiológicas del agua para consumo humano deben enmarcarse dentro de los valores de 1 Unidad Formadora de Colonia (UFC) ó 1 microorganismo en $100 \mathrm{~cm}^{3}$ de muestra, el punto número 1 de osmosis se 
obtuvieron 250 UFC se presencia de aerobios mesófilos los cuales sobrepasan lo estipulado por la normatividad ambiental [7].

De acuerdo a los análisis encontrados, se evidenció que el equipo de ósmosis generaba posibles alteraciones del agua por el tiempo de uso. Para evidenciar ello, se realizó un ensayo con un equipo de ósmosis nuevo, generando los siguientes resultados que se muestran en la tabla III:

Tabla III: Resultado análisis fisicoquímico y microbiológico del equipo nuevo de ósmosis.

\begin{tabular}{|c|c|c|c|}
\hline PARÁMETROS & $\begin{array}{l}\text { PUNTO 1. ENTRADA } \\
\text { ÓSMOSIS }\end{array}$ & $\begin{array}{c}\text { PUNTO 2. ÓSMOSIS } \\
\text { CHAQUETA }\end{array}$ & NORMATIVIDAD AMBIENTAL \\
\hline MUESTRA & $200 \mathrm{~mL}$ & $200 \mathrm{~mL}$ & - \\
\hline CAUDAL & $0.6 \mathrm{~L} / \mathrm{s}$ & $0.1 \mathrm{~L} / \mathrm{s}$ & $1.5(\mathrm{QMH})$ \\
\hline PH IN SITU & 7 & 7 & \\
\hline pH LABORATORIO & 6.23 & 6,23 & $5-9$ \\
\hline TEMPERATURA & $26^{\circ} \mathrm{C}$ & $24^{\circ} \mathrm{C}$ & $<40^{\circ} \mathrm{C}^{* *}$ \\
\hline DQO & $30 \mathrm{mg} / \mathrm{L}$ & $0 \mathrm{mg} / \mathrm{L}$ & $200 \mathrm{mg} / \mathrm{L}$ \\
\hline TURBIDEZ & 0.75 NTU & $0.53 \mathrm{NTU}$ & $<5$ unidades \\
\hline COLOR & O PtCo & O PtCo & 75 unidades \\
\hline CONDUCTIVIDAD & $19,45 \mu \mathrm{S} / \mathrm{cm}$ & $23,09 \mu \mathrm{S} / \mathrm{cm}$ & $<1500 \mu \mathrm{S} / \mathrm{cm}$ \\
\hline ALCALINIDAD & $0 \mathrm{mg} / \mathrm{L}$ & $0 \mathrm{mg} / \mathrm{L}$ & $100 \mathrm{mg} / \mathrm{L}$ \\
\hline DUREZA & _> $100 \mathrm{ppm}$ & _>100 ppm & $160 \mathrm{mg} / \mathrm{L}$ \\
\hline $\begin{array}{l}\text { CARBONO ORGÁNICO } \\
\text { TOTAL }\end{array}$ & - & $27 \mathrm{mg} / \mathrm{L}$ & Análisis y soporte \\
\hline Aerobios Mesófilos & $0 \mathrm{ufc} / \mathrm{ml}$ & $0 \mathrm{ufc} / \mathrm{ml}$ & $100 \mathrm{UFC} / 100 \mathrm{ml}$ \\
\hline Clostridium sp & $0 \mathrm{ufc} / \mathrm{ml}$ & $0 \mathrm{ufc} / \mathrm{ml}$ & $0 \mathrm{UFC} / 100 \mathrm{ml}$ \\
\hline Hongos y Levaduras & $0 \mathrm{ufc} / \mathrm{ml}$ & $0 \mathrm{ufc} / \mathrm{ml}$ & $20 \mathrm{UFC} / 100 \mathrm{ml}$ \\
\hline Coliformes fecales y totales & $0 \mathrm{ufc} / \mathrm{ml}$ & $0 \mathrm{ufc} / \mathrm{ml}$ & $0 \mathrm{UFC} / 100 \mathrm{ml}$ \\
\hline Bacillus Sp & Ausencia & Ausencia & Ausentes \\
\hline
\end{tabular}

Fuente: Autores

De acuerdo a los resultados arrojados en la toma de muestra que se observan en la tabla III y según Resolución 0631 Artículo 8 Página 7 Valores límites máximos permisibles en los vertimientos puntuales de ARnD.; Decreto 1594/84 Artículos 38, 72 y 73. Vigente aun porque el Decreto 3930/2010 empezará a ser vigente a partir de enero de 2016.; Decreto 475/98 Artículo 8 Literal b) y Artículo 36 Criterios químicos de la calidad de agua potable y criterios de calidad organolépticas y físicas del agua segura.; Resolución 151/2001 Comisión de Regulación de Agua Potable y
Saneamiento Básico [8]; el filtro nuevo está removiendo eficientemente DQO no así con la conductividad pues esta aumenta esto debido a que probablemente el filtro está añadiendo partículas disueltas como se percibe en la turbiedad, lo que afecta aceptabilidad del agua y puede generar preocupación por la calidad. También puede indicar una gran propensión a la generación de subproductos si se involucran procesos de desinfección. En otros casos, por ejemplo, partículas rojizas-cafés (hierro) o rojizas-negras (manganeso) pueden estar visibles cuando el agua sale del grifo. Estas 
partículas de hierro y/o manganeso pueden provenir de tubos corroídos o del mismo abastecimiento de agua ${ }^{4}$ Las partículas se forman debido a que el oxígeno en el sistema de plomería está oxidando y precipitando el hierro y el manganeso. Si el agua está clara cuando sale del grifo, pero las partículas se forman y se acumulan después de que el agua ha estado estancada por un rato, el hierro y/o manganeso están en el suministro del agua. Se disuelven en el agua y permanecen invisibles hasta que se oxidan y se precipitan. Algunas veces el agua del grifo tiene un color rojizo. Esto es causado por el hierro coloidal-hierro que no forma partículas lo suficientemente grandes para precipitarse [8].

\section{Conclusiones}

De acuerdo a las variaciones de las características fisicoquímicas y microbiológicas el agua no es apta para recircular, por lo cual es necesario un proceso posterior para volver el agua tratable, a pesar de estas alteraciones la autoclave cumple los parámetros establecidos para los ciclos de esterilización el cual no se vio afectado en ninguna de las pruebas.

Comparando los datos obtenidos en los análisis microbiológicos realizados se evidencio que el agua en los diferentes puntos cumple con los parámetros que están establecidos por la resolución 2115 de22 de junio del 2007 y la NTC 8013; de igual forma se pudo establecer que el agua tratada con osmosis muestra en ciertos parámetros rangos fuera del máximo establecidos por dichas normas demostrando que con el paso del tiempo es posible que el equipo de osmosis vaya disminuyendo su eficiencia

Se realizó comparación de las características físico químicos y microbiológicas del agua utilizando la autoclave a vapor de una institución de salud y el de la empresa estudiada utilizado especialmente para el proyecto con el cual se afirma que no presenta alteración y ambos equipos garantizan el proceso de esterilización.

Ante la posibilidad de un biofilm (Contaminación del agua) en el filtro de agua de osmosis se realizan nuevas pruebas utilizando un filtro de agua de osmosis nuevo, del cual los resultados arrojados no presentaron alteraciones según los parámetros ambientales y microbiológicos.

\section{Agradecimientos}

A la empresa Stericlinic S.A.S por su valiosa colaboración para la obtención de los datos que sirvieron para la elaboración y desarrollo del proyecto.

\section{Referencias.}

[1]. Acosta-Gnass SI, Stempliuk VdA. Manual De Esterilización Para Centros De Salud. [Online].; Washington D.C 2008. Available from: HYPERLINK "http://www.inicc.org/guias/amrmanual-esterilizacion.pdf" http:// www.inicc.org/guias/amr-manualesterilizacion.pdf

[2] F. Canestro Márquez et al. "TCAE en el servicio de esterilización", Editorial Vértice, España, 2007

[3]. S.I Acosta-Gnass, V.DA Stempliuk. "Manual de esterilización para Centros de Salud", Organización Panamericana de la Salud, Washington D.C, 2008.

[4]. M. D. M Serra Zamora. "Guía para el manejo de la autoclave en la central de esterilización del hospital universitario de Ceuta”, Instituto Nacional de gestión sanitaria, España, 2013.

[5]. J.A Orellana. "Características del agua potable" en Ingeniería Sanitaria- UTN - FRRO, Universidad Tecnológica Nacional, Argentina, 2005.
Julio - Dic. 2017 ISSN 0122-820X E-ISSN 2422-5053 PP: 59-66 
Julio - Dic. 2017

ISSN 0122-820X

E-ISSN 2422-5053

PP: 59-66

[6] A. Borja Hernani et al. "Manual de desinfección y esterilización hospitalaria", Ministerio de Salud, Perú, 2002.

[7] Instituto Colombiano de Normas Técnicas y Certificación - ICONTEC. Norma Técnica NTC 813 (Segunda actualización). [En línea]. Disponible en: http://ingenieria.udea.edu.co/isa/ normas_decretos/TEXTO $\% 20 \mathrm{NTC} \% 20$ 813\%20AGUA\%20POTABLE.pdf

[8] Ministerio de la Protección Social. Resolución 2115, 2007. [En línea]. Disponible en: http://www.ins.gov.co/ tramites-y-servicios/programas-decalidad/Documents/resolucion $\% 20$ 2115\%20de \%202007,MPS-MAVDT. pdf 